EPJ Web of Conferences 33, 03004 (2012)

DOI: $10.1051 /$ epjconf/20123303004

(C) Owned by the authors, published by EDP Sciences, 2012

\title{
Concentrating solar power in Europe, the Middle East and North Africa: achieving its potential
}

R. Pitz-Paal ${ }^{1, \mathrm{a}}$, A. Amin², M. Bettzüge ${ }^{3}$, P. Eames ${ }^{4}$, F. Fabrizi ${ }^{5}$, G. Flamant ${ }^{6}$, , F. Garcia Novo ${ }^{7}$, J. Holmes ${ }^{8}$, A. Kribus ${ }^{9}$, H. van der Laan $^{10}$, C. Lopez ${ }^{11}$, P. Papagiannakopoulos ${ }^{12}$, E. Pihl ${ }^{13}$, P. Smith ${ }^{14}$ and H-J. Wagner ${ }^{15}$.

${ }^{1}$ DLR, Porz-Wahnheide, Linder Hohe, 51147 Koln, Germany

${ }^{2}$ Helwan University, Faculty of Engineering, 8 Giza Street, Giza 12211, Egypt

${ }^{3}$ University of Cologne, Institute of Energy Economics (EWI), Albertus-Magnus-Platz, D-50923, Cologne, Germany

${ }^{4}$ Loughborough University, Centre for Renewable Energy Systems Technology, Holywell Park,

School of Electronic, Electrical and Systems Engineering, Loughborough LE11 3TU, UK

${ }^{5}$ ENEA - UTRINN - STD (Solar Thermodynamic Laboratory), Via Anguillarese 301, 00123, Rome, Italy

${ }^{6}$ PROMES-CNRS, 7 rue du Four Solaire, Odeillo, 66120 Font Romeu, France

${ }^{7}$ University of Seville, C/ San Fernando 4, 41004 Seville, Spain

${ }^{8}$ EASAC, Leopoldina, Postfach 110543, 06019 Halle (Saale), Germany

${ }^{9}$ Tel Aviv University, School of Mechanical Engineering, Faculty of Engineering, Tel Aviv 69978, Israel

${ }^{10}$ Universities of Leiden and Utrecht, Schoener 18, 3961 KZ Wyk by Duurstede, Netherlands

${ }^{11}$ CIEMAT, Avda. Complutense, 40-28040 Madrid, Spain

${ }^{12}$ University of Crete, Department of Chemistry, 71003 Heraklion, Crete, Greece

${ }^{13}$ Chalmers University of Technology, Department of Energy and Environment, SE-412 96 Goteborg, Sweden

${ }^{14}$ University College Dublin, Electricity Research Centre, Engineering \& Materials Science Centre, Belfield, Dublin 4, Ireland

${ }^{15}$ Ruhr-Universitat Bochum, 44780 Bochum, Germany

\begin{abstract}
Concentrating solar power (CSP) is a commercially available renewable energy technology capable of harnessing the immense solar resource in Southern Europe, the Middle East and North Africa (the MENA region), and elsewhere. This paper summarises the findings of a study by the European Academies Science Advisory Council which has examined the current status and development challenges of CSP, and consequently has evaluated the potential contribution of CSP in Europe and the MENA region to 2050. It identifies the actions that will be required by scientists, engineers, policy makers, politicians, business and investors alike, to enable this vast solar resource to make a major contribution to establishing a sustainable energy system. The study concludes that cost reductions of 50-60\% in CSP electricity may reasonably be expected in the next 10-15 years, enabling the technology to be cost competitive with fossil-fired power generation at some point between 2020 and 2030. Incorporation of storage delivers added value in enabling CSP to deliver dispatchable power. Incentive schemes will be needed in Europe and MENA countries to enable this point to be achieved. Such schemes should reflect the true value of electricity to the grid, effectively drive R\&D, and ensure transparency of performance and cost data.
\end{abstract}

\section{Introduction}

Concentrating solar power sits alongside photovoltaic electricity generation as a commercially available renewable energy technology capable of harnessing the immense solar resource in Southern Europe, the Middle East and North Africa (the MENA region), and elsewhere. In concentrating solar power (CSP) a high temperature heat source is created by concentrating the sun's rays to produce electricity in a thermodynamic cycle.

This paper summarises the findings of a study by the European Academies Science Advisory Council (EASAC, established in 2001 by the national academies of the EU member states to advise European policy makers on the science underpinning key EU policy issues: www.easac.eu ) which has examined the current status and development challenges of CSP, and consequently has evaluated the potential contribution of CSP in Europe and the MENA region to 2050 [1]. It identifies the actions that will be required by scientists, engineers, policy makers, politicians, business and investors alike, to enable this vast solar resource to make a major contribution to establishing a sustainable energy system.

In 2011, as illustrated in Figure 1, there was $1.3 \mathrm{GW}$ of CSP operational worldwide, 2.3GW under construction, and $31.7 \mathrm{GW}$ planned (derived from [2-6]). Spain has been the leading exponent of CSP in Europe to date. 


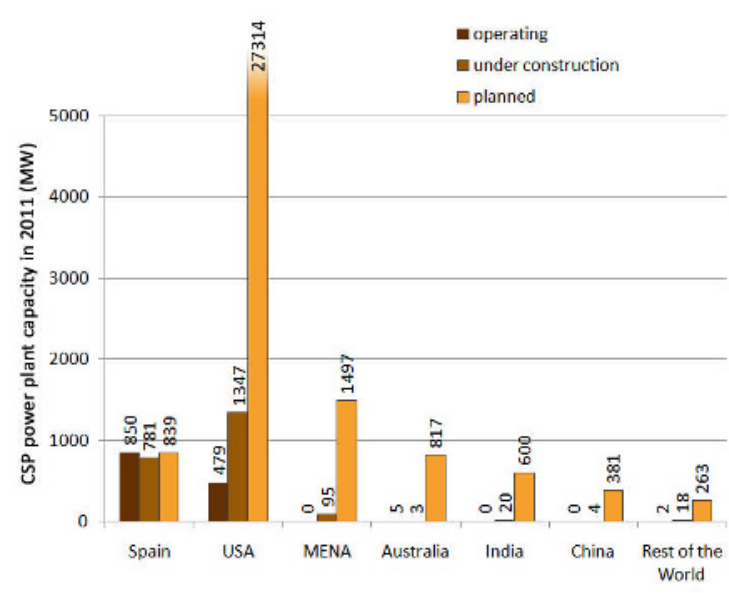

Fig. 1: Worldwide distribution of CSP plants that are operational, under construction and planned

\section{Current status and technological developments}

A CSP plant comprises four main sub-systems as shown schematically in Figure 2: concentrating system, solar receiver, storage and/or supplementary firing (labelled 'back-up system' in the figure), and power block. They are linked together by radiation transfer or fluid transport.

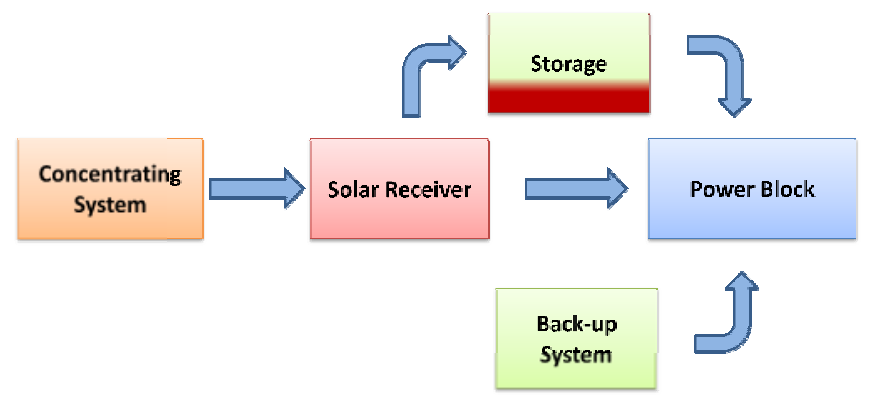

Fig. 2: Main components and sub-systems of a CSP plant including storage

An important inherent option for CSP power plants is to incorporate thermal storage, enabling power to be generated when the sun is not shining, and contributing to CSP's distinctive (in comparison to many other renewable electricity generation technologies) ability to provide dispatchable power. As shown in Figure 3, there are four main CSP technology families there are four main CSP technology families that can be classified according to the way they focus the sun's rays and the receiver technology.

CSP generating costs are currently similar to offshore wind, slightly above photovoltaics (PV), and around twice those of a midload coal-fired plant (without carbon capture and storage) and onshore wind [1]. However, studies have consistently pointed to the potential to reduce CSP generating costs by around $60 \%$ (e.g. [7]), of which around half should come from scaling up and volume production, the rest from technological innovations.

Horizontal technological improvements are anticipated, potentially providing benefits across the families of CSP technologies. For mirrors these improvements include increasing reflectivity to $95 \%$ (by developing thinner front glass), anti-soiling and hydrophobic coatings on glass (to prevent dust deposition and reduce cleaning requirements), front surface aluminized reflectors, and polymer reflectors. Interrelated technology breakthroughs are expected in heat transfer fluids, storage media and thermodynamic cycles:

- Heat transfer fluids: superheated steam, new molten salts (with low melting temperature and higher working temperatures), nano-fluids, pressurized air (mainly development of new solar receivers), and circulating particles.

- Storage: phase change materials for direct steam generation, high temperature storage for gas cycles, compact heat storage (chemical reactions), and heat transfer concepts.

- Thermodynamic cycles: supercritical steam or carbon dioxide cycles, air Brayton cycles and combined cycles (for tower technology). 


\begin{tabular}{|c|c|c|}
\hline $\begin{array}{ll}\text { Receiver } & \text { Focus type } \\
\end{array}$ & Line focus & Point focus \\
\hline $\begin{array}{l}\text { Fixed } \\
\text { Stationary receiver that remains } \\
\text { mechanically independent of the } \\
\text { concentrating system. The attainable } \\
\text { working temperature depends of the } \\
\text { concentration ratio. }\end{array}$ & $\begin{array}{l}\text { Linear Fresnel reflector (ifR) } \\
\text { Cunved } \\
\text { niros }\end{array}$ & Central receiver \\
\hline $\begin{array}{l}\text { Tracking / aligned } \\
\text { The receiver moves together with the } \\
\text { concentrating system. Mobile receivers } \\
\text { collect more radiation energy than } \\
\text { corresponding fixed receivers. }\end{array}$ & Parabolic trough & Parabolic dish \\
\hline
\end{tabular}

Fig. 3: The four main CSP technology families (after [8])

In order to realise these technology breaktroughs and associated cost and efficiency improvements, it is essential to coordinate the different research, development and demonstration efforts with a market incentivation that favours cost reduction by innovation over cost reduction by mass production of state of the art technology options. Research without the chance to implement the technology in the market, and to improve and adapt it over a couple of technology generations, has a high risk of failure in a competitive market.

Increased research funding and a stronger integration of fundamental and applied research, together with demonstration programmes and market incentives, are required to speed up the innovation cycle. Fundamental research on new materials, heat transfer fluids, and coatings is needed, and integrated programmes should enable smooth progression of promising technologies from laboratory scale prototype systems to pilot plants and demonstrations units. Results of the individual phases should be independently evaluated and benchmarked with respect to their impact on system cost targets before starting on the next phase.

\section{Achieving economic competitiveness}

An alternative approach to estimating future potential for cost reduction is to use well-established 'learning curve' effects, which are based on observations for technologies more generally that their cost reduces by a characteristic percentage for each doubling of installed capacity (hence, the 'learning rate' is defined as the percentage reduction in costs for each doubling of installed capacity). The left hand side of Figure 4 indicates the cumulative CSP capacity needing to be installed to achieve a 50\% generating cost reduction for learning rates of 10 and $20 \%$ which span the range typically achieved by new renewable energy technologies. Taking a $50 \%$ cost reduction as (broadly) that necessary to achieve breakeven with fossil-fired generation (at current fossil fuel prices and without carbon capture and storage), around 9GW of CSP will need to be installed to achieve cost competitiveness if the learning rate is $20 \%$, and $100 \mathrm{GW}$ if it is $10 \%$.
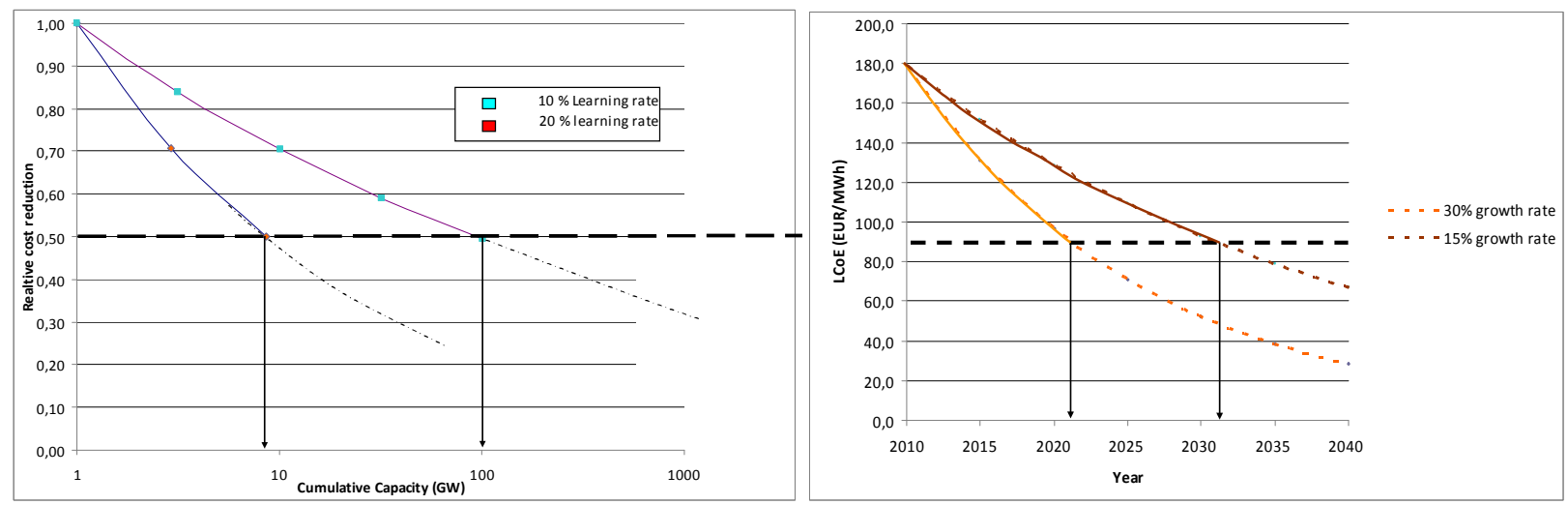

Fig. 4: Left: Relative cost of CSP technology as a function of the cumulative installed capacity for learning rates of 10 and $20 \%$; Right: Development of LCoE (Lifetime Cost of Electricity) over time for CSP systems installed at $15 \%$ (low) and 30\% (high) growth rates per year (based on a learning rate of $15 \%$ ). 
The right hand side of Figure 4 illustrates the potential implications of a learning rate of 15\%, i.e. in the middle of this range and close to the estimate of 14\% of [9], for when CSP may reach a 50\% cost reduction. Starting from a current CSP installation rate of around $500 \mathrm{MW}$ per year, and assuming a growth rate in CSP installations of 15\% (low) and 30\% (high) per year, results in CSP achieving a $50 \%$ cost reduction at some point in the $2020 \mathrm{~s}$, enabling it to be cost competitive with other generating options.

The learning rate and the growth rate of installed CSP capacity are key determinants of when CSP will be cost competitive with other technologies, and the cumulative subsidy required to achieve that point. The ranges of figures selected in this analysis are based on expert estimates and opinion, and have not been verified by actual data which are not available. It is therefore strongly recommended that mechanisms are put in place that enforce a transparent monitoring of installation costs, and the rate of CSP technology capacity increases, to enable estimates of the learning rate to be refined.

It is not just the cost of CSP generation which determines its economic competitiveness, but its value, which has three components:

- the value of the kWh's of electrical energy generated by the plant, which will vary over time in a competitive electricity market, reflecting the availability and cost of electricity from other sources;

- the contribution that the CSP plant makes to ensuring that generating capacity is available to meet peak electricity system demand; and

- the 'services' provided by the plant in helping the electricity transmission system operator to balance supply and demand in the short term (typically, on timescales of seconds and minutes).

Potentially, incorporation of thermal storage in a CSP plant can be beneficial to all three components of value. In relation to the first component, system simulations conducted in connection with the study [10] indicate that as the solar share rises in an energy system there is increasingly value in shifting generation to the evenings when the sun is not shining, and hence an incentive to install CSP plants with thermal storage. The availability of such plants in the system means that higher penetrations of solar power can be achieved overall and is an important consideration, beyond just generating costs, in determining the optimum mix of CSP and PV.

With regard to the second component of value, the provision of generating capacity to meet peak electricity system demand, CSP with storage can contribute to meeting peak system loads and can provide back-up capacity to cover variable renewable sources. Incorporation of supplementary firing will further increase the capability of the CSP plant to provide capacity at the system peak, though the efficiency of fossil-fuel use for such supplementary firing is likely to be significantly lower than if it is used in a combined cycle power plant. The value of providing capacity to meet the system peak demand will depend on the system, so its quantification needs to be informed by system models.

Turning to the third component, the value of thermal energy storage in enabling the CSP plant to deliver grid services, CSP with storage can provide spinning reserves, being able to ramp up power if operating at part-load in less than 30 minutes by drawing on the stored heat (the rate of ramping is limited by the thermal inertia of the equipment). Ramping down is quicker: on timescales of around 15 minutes by diverting heat to storage. This is used in Spain to deliver, on demand, 30\% power ramps in less than an hour, enabling the plant to be considered dispatchable by the grid operator REE.

In discussing the services provided by a CSP plant in helping the electricity system operator to address short-term supply-demand imbalances, consideration must be given to the potential 'negative value' arising from transients during partly cloudy days. Inclusion of at least three hours storage in the CSP plant enables the substantial thermal inertia provided by the storage medium to be used to dampen any resulting steam temperature/pressure gradients at the power block inlet on such days.

Incorporation of thermal storage in a CSP plant results in significant added value compared to PV plants or CSP without storage, and means that the plants can match most of the dispatchability characteristics of a mid-load fossil-fired plant.

\section{Prospects to 2050}

In considering the potential role of CSP in Europe towards 2050, the EU's objective of reducing greenhouse gas emissions by 8095\% by 2050 is a key parameter. Re-affirmed by the European Council in February 2011, this objective requires the EU's electricity system to achieve essentially zero emissions of greenhouse gases by 2050 [11].

The 2050 generating mix may include nuclear power and fossil-fired power stations incorporating carbon capture and storage. But on-going public concerns about nuclear power, exacerbated by the Fukushima accident in Japan, have led some countries such as Germany to exclude it from consideration. Carbon capture and storage on fossil-fired power stations remains essentially unproven at commercial scale, with questions remaining as to whether sufficient safe storage sites, acceptable to the public and regulators, can be found. And it locks in Europe's exposure to fossil fuel price escalation and volatility. 
Variable renewable sources such as wind, solar PV and marine energy will be required to play a major role in Europe's 2050 electricity system, but their variability will bring challenges of balancing supply and demand. An integrated European grid and market, together with demand management may go some way to meeting these challenges, but additional system storage capacity may be needed, and controllable renewable sources will be at a premium. Such sources include hydro and geothermal energy - but in both cases natural resources in Europe are limited - and CSP with storage, for which natural resources far outstrip anticipated electricity demand when account is taken of CSP potential in the neighbouring MENA region.

Whereas many forecasts anticipate limited, or no, growth in European electricity demand to 2050, in the MENA region population growth and economic development are expected to result in a rapid increase in electricity demand, potentially reaching similar overall levels to the EU by 2050 (for example [12]). International initiatives to limit global warming emphasise that such development should follow a sustainable path, putting an onus on maximising the use of indigenous renewable resources: the solar resource, of course, being dominant in the MENA region. However, as such renewable capacity is currently significantly more expensive than the fossil alternative, and given their economic starting point, MENA countries will require foreign assistance to follow such a low-carbon path.

It is not a shortage of sunshine in Southern Europe and the MENA region which will constrain CSP's contribution but other factors, particularly:

- CSP's generating costs in relation to alternative technologies, and the values of $\mathrm{CO}_{2}$ mitigation and of CSP generation compared to alternatives;

- physical constraints on the installation of CSP generating capacity due to the availability of land, water, manufacturing capacity, skilled labour etc;

- physical and operational constraints on the transmission of electricity across Europe and the MENA region to balance supply and demand; and

- considerations of security of supply, particularly the comparative vulnerabilities inherent in different energy vectors when imported from other countries.

The previous section has discussed the sensitivity of generation costs to learning rates and the consequent implications for cumulative subsidies required to achieve economic competitiveness. For example, if today $60 \%$ of the CSP capital cost needs to be subsidised (assumed for simplicity as a grant) but only a 10\% subsidy is needed when CSP generating costs are halved, then the cumulative subsidy to achieve a halving of costs is $€ 6.5$ billion for a learning rate of $20 \%$ (corresponding to an installed capacity of $9 \mathrm{GW}$ ), and $€ 61$ billion if it is only $10 \%$ (corresponding to an installed capacity of $100 \mathrm{GW}$ ). Two recent estimates of the total incentive payments needed to achieve cost parity fall within this range of cumulative subsidies: Ummel and Wheeler [13] estimate it at US\$ 20 billion (corresponding to $20 \mathrm{GW}$ of CSP), Williges et al [14] at $€ 43$ billion for their baseline case (corresponding to 157 $\mathrm{GW}$ of CSP).

With regard to physical constraints, water availability is an issue for CSP, particularly in desert regions, and there is a need for further development of dry cooling systems which minimise the associated generating efficiency penalty. Plenty of potentially suitable land for CSP installations exists, particularly in the MENA region, but land acquisition, planning permissions etc take time and might at some point constrain high rates of development of CSP, particularly in Southern Europe.

Achieving an essentially zero carbon electricity system in Europe by 2050, will require the replacement of much of the existing generating capacity over the intervening period. Similarly, meeting the MENA region's anticipated expansion in electricity supply will require large and sustained investments in new generating capacity. The availability of the required manufacturing capacity for a major expansion of CSP is appropriately considered in this context, particularly as many of the plant components such as turbines, heat exchangers, piping etc are common to many of the candidate technologies. Significant increases in, and shifts of, manufacturing capacity will be required whichever generating mix is chosen. While CSP is more material intensive in its construction that fossilfired plants (primarily in commonplace materials such as steel, glass and concrete), given the levels of production of these materials in the economy more generally, it seems unlikely that their availability will prove to be an insurmountable constraint on CSP expansion. A sustained and rapid growth of CSP in Europe and the MENA region would require coordinated efforts to enable the associated re-deployment and re-skilling of a substantial workforce, but it is instructive to note that in a five-year period the renewable energy industry in Europe increased its workforce from 230,000 to 550,000 [11].

It is generally considered that a high voltage direct current (HVDC) transmission grid needs to be built as a 'back bone' or 'super-highway' across Europe and the MENA region to augment existing high voltage alternating current (HVAC) transmission and distribution systems. Modern HVDC lines can limit transmission losses over $3000 \mathrm{~km}$ to around $10 \%$. Transfer of electrical power over such distances is an impractical proposition for HVAC lines where the losses would be nearer to 50\% [15]. In addition, HVAC grids will need to be reinforced and 'smart' grid technologies will be widely deployed. The current limitations of Europe's electricity grid are well-recognised in the EU's energy strategy which aims to secure the grid reinforcements necessary for the effective 
functioning of the EU market and the trans-national transfers of bulk electricity associated with geographical diversity as a mechanism for matching supply and demand for renewable energy sources [16].

Increasing security of supply of energy is a key concern of EU energy policy. To the extent that CSP capacity is located in Southern Europe, it contributes positively to increasing supply security as it reduces the need for energy imports (currently standing at over $50 \%$ of the EU's energy use, mainly for fossil fuels). The security of supply issues associated with Europe importing CSP electricity from the MENA region are not so clear cut. Whereas interruption of power supplies can cause significant harm, unlike fossil fuels and uranium, an interruption in the supply of electricity would represent an unrecoverable loss of revenue for supply countries, because electricity cannot be stored, and would likely harm exporting countries more than the supply interruption would harm Europe [17]. Diversification of supply sources and routes can help to mitigate the risks of supply interruptions due to terrorism or political interference, and currently there is a substantial reserve of fossil-fired capacity.

Import of CSP electricity would enable reduction of the imports of fossil fuels which constitute a major risk to Europe due to the possibility of supply interruptions, and the economic consequences of price volatility and potential sustained future price rises if the world does not take coordinated action to reduce fossil fuel dependence [11]. Integration of energy markets with neighbouring countries is a particular EU initiative which should help to mitigate risks from CSP imports [16, 18].

\section{Three key challenges}

The solar resource in Southern Europe is such that CSP could provide a useful contribution to achieving Europe's aim of a zerocarbon electricity system by 2050. Solar resources in the MENA region are even better, and far larger. Once CSP achieves cost parity with fossil-fired generation, estimated to be at some point between 2020 and 2030 though potentially earlier in particular circumstances, these resources have the potential to transform the system of electricity generation in Europe and the MENA region. However, three key challenges will need to be overcome if this transformation is to be achieved.

The first challenge is to move towards, and in time to achieve, cost parity of CSP and fossil-fired generation. Around half of the anticipated reductions in CSP generating costs are expected to come from technology developments, and the other half from economies of scale and volume production. The study has identified the most promising areas of scientific and technological development to realise cost reductions. Well-designed incentive schemes will be needed, which reflect the real, time-varying value of generation so that CSP plants are appropriately designed, and which effectively drive research and development activities. Schemes need to ensure that new CSP technology innovations can progress rapidly from the laboratory to pilot and demonstration scales, and to commercial application.

Incentive schemes may be specific to particular technologies (for example, differentiating between CSP and PV), or may give more generic support to increasing the installed capacity of low-carbon technologies while also supporting technology specific research, development and demonstration. In either case, the total amount of subsidy that will be needed to achieve cost parity will depend crucially on how quickly costs reduce as installed capacity increases. Incentive schemes should ensure that cost data are made available so that the learning rate, and its underlying drivers, can be established and monitored, and consequently energy strategies and incentive schemes can be adjusted as appropriate.

In the medium term, CSP's ability to support the system integration of variable renewable electricity sources suggests that its further support should not be determined solely by its short-term competitiveness with PV systems. CSP and PV may prove to be complementary technologies in harnessing the solar resource, and it is appropriate to continue to support both technologies at the present time.

CSP technologies are consistent with a high share of local value creation, which with appropriate investments in skills and manufacturing facilities may be expected to increase over time. This local benefit is more pronounced than for other renewable technologies such as PV, and supports economic development, particularly in countries with increasing industrialisation, creating local jobs, wealth and expertise, making CSP a particularly appropriate technology for the MENA region.

The second challenge is to establish the grid connections and market mechanisms that will enable the integration of solar power in Europe and in the MENA region. If substantial amounts of CSP electricity are to be exported from the MENA region to Europe, then large investments will need to be made in grid connections between MENA countries and Europe, and in HVDC lines in Europe to transport electricity to demand centres.

Further system simulation studies should be undertaken, including the use of high resolution and (ideally) stochastic power system models, to look at interaction effects for different shares of renewable energy sources at EU, MENA and EU-MENA levels of power system integration. Understanding from these studies, together with data on the learning rates of CSP and PV technologies, should be used to guide the development of the optimal mix to harness solar resources, and the necessary transmission infrastructure. 
$2^{\text {nd }}$ European Energy Conference

The third challenge relates to the development of CSP in the MENA region as a potentially significant component of initiatives to support low-carbon economic development and political progress in the region, while addressing security of supply concerns if Europe were to rely heavily on solar power from the MENA region. Given the rapidly increasing demand for electricity in MENA countries, much of the electricity generated by CSP plants in the MENA region over the short to medium timescale may, and should, be expected to be used locally rather than exported to Europe, thus avoiding the construction of fossil-fired capacity in the MENA region. Financing schemes, and associated political agreements between the EU and MENA countries, will be needed to enable these short to medium timescale developments. Without financial commitments in the order of $€$ billions from Europe, renewable energy technologies, including CSP, are unlikely to develop quickly in the MENA region

Looking towards 2050, if investments in CSP capacity in the MENA region are sufficient, there is the potential for major exports of electricity to Europe. It is possible that solar-generated hydrogen and syngas exports may also play a role. The closer economic and social integration of the EU and MENA region anticipated by the Barcelona Process, the Deauville Partnership etc will be critical in ensuring that security of supply concerns can be allayed. Imports of solar electricity from the MENA region would lower dependence on imports of fossil fuels from that region, and other regions too.

The rationale for Europe to support CSP deployment in the MENA region derives in part from its commitments to support sustainable economic development in the region, and is twofold. Firstly, CSP is an attractive and easily integrated option to limit $\mathrm{CO}_{2}$ emissions resulting from the increased energy consumption associated with population growth and economic development in this region. Secondly, local suppliers can undertake a substantial portion of the activities needed to design, build and operate CSP plants, bringing regional development and employment benefits, and consequently contributing to the development of stable societies.

A coordinated approach is needed, simultaneously addressing the different bottlenecks (investment protection, energy policy incentives, R \& D etc), and identifying options which lower the barriers to entry for other actors. For this purpose, a transformation process needs to be designed and supported scientifically over a long period. Scientific academies in Europe and the MENA region can play a useful role in supporting this process.

\section{References}

1. EASAC. Concentrating solar power: its potential contribution to a sustainable energy future. EASAC policy report 16. (2011). http://www.easac.eu/home/reports-and-statements/detail-view/article//concentratin.html

2. California Energy Commission. Large solar energy projects (2010) http://www.energy.ca.gov/siting/solar/index.html

3. CSP Today. CSP world plant locations (2011) www.csptoday.com/usa/csp-world-mapmainen.shtml

4. Greentechmedia. US CSP project tracker (2011) http://www.greentechmedia.com/images/wysiwyg/researchblogs/USCSPProjectTracker.pdf

5. Protermosolar. Mapa de la Industria Solar Termoélectrica en España (Map of the Solar Thermal Power Industry in Spain (2011) http://www.protermosolar.com/mapa.html

6. US Bureau of Land Management. Pending Arizona BLM solar projects (2011) http://www.blm.gov/az/st/en/prog/energy/solar/pend-solar.html

7. R. Pitz-Paal, J. Dersch, B. Milow, 2005. European Concentrated Solar Thermal Road-Mapping (ECOSTAR): roadmap document. SES6-CT-2003-502578 (2005) http://www.promes.cnrs.fr/uploads/pdfs/ecostar/ECOSTAR.Summary.pdf

8. International Energy Agency (IEA). Technology Roadmap: Concentrating Solar Power (2010) http://www.iea.org/papers/2010/csp_roadmap.pdf

9. F. Trieb. SOKRATES-Projekt: Solarthermische Kraftwerkstechnologie für den Schutz des Erdklimas. DLR (2004) http://www.dlr.de/tt/Portaldata/41/Resources/dokumente/institut/system/projects/AP1_3_ATHENE.pdf

10. S. Nagl, M. Fürsch, C. Jägemann, M. Bettzüge. The economic value of storage in renewable power systems - the case of thermal energy storage in concentrating solar power plants. EWI Working Paper No. 11/08 (2011) http://www.ewi.unikoeln.de/publikationen/working-paper/

11. European Commission. A Roadmap for moving to a competitive low carbon economy in 2050. Communication from the Commission, 8.3.2011, COM(2011) 112 final (2011) http://eurlex.europa.eu/LexUriServ/LexUriServ.do?uri=COM:2011:0112:FIN:EN:PDF

12. DLR. Concentrating solar power for the Mediterranean region. Final report for German Federal Ministry for the Environment, Nature Conservation and Nuclear Safety (2005) http://www.dlr.de/tt/Portaldata/41/Resources/dokumente/institut/system/publications/MED-CSP_complete_study.pdf

13. K. Ummel, D. Wheeler. Desert power: the economics of solar thermal electricity for Europe, North Africa and the Middle East. Center for Global Development, Working paper no. 156 (2008) www.cgdev.org/files/1417884_file_Desert_Power_FINAL_WEB.pdf

14. K. Williges, J. Lilliestam, A. Patt. Energy Policy 38, 3089 (2010)

15. DLR. TRANS-CSP: Trans-Mediterranean interconnection for Concentrating Solar Power. Final report for study commissioned by the Federal Ministry for the Environment, Nature Conservation and Nuclear Safety, Germany (2006) $\underline{\text { www.dlr.de/tt/trans-csp }}$ 
16. European Commission. Energy 2020: A strategy for competitive, sustainable and secure energy. Communication from the Commission COM(2010) 639 final (2010) http://www.energy.eu/directives/com-2010-0639.pdf

17. IIASA. Expanding solar energy in North Africa to achieve climate targets. IIASA Policy Brief No. 7 (2009) http://www.iiasa.ac.at/Admin/PUB/policy-briefs/pb07-web.pdf

18. European Commission. On security of energy supply and international cooperation - "The EU Energy Policy: Engaging with Partners Beyond our Borders". Communication from the Commission to the European Parliament, the Council, the European Economic and Social Committee and the Committee of the Regions, COM(2011) 539 final. (2011)

http://ec.europa.eu/energy/international/security_of_supply/doc/com_2011_0539.pdf 